Original Article

\title{
Immediate effects of kinematic taping on lower extremity muscle tone and stiffness in flexible flat feet
}

JoONG-SAN WANG ${ }^{1)}$, Gi-MAI UM ${ }^{2}{ }^{*}$, JunG-HyUn $\mathrm{CHOI}^{3)}$

1) Department of Physical Therapy, Howon University, Republic of Korea

2) Department of Physical Therapy, Yeoju Institute of Technology: 338 Sejong-ro, Yeoju-si, Gyeonggi-do, Republic of Korea

3) Department of Physical Therapy, Institute for Elderly Health and Welfare, Namseoul University, Republic of Korea

\begin{abstract}
Purpose] This study aimed to examine the immediate effects of kinematic taping on the tone and stiffness in the leg muscles of subjects with flexible flat feet. [Subjects and Methods] A total of 30 subjects, 15 in the kinematic taping and 15 in the sham taping group, were administered respective taping interventions. Subsequently, the foot pressure and the tone and stiffness in the tibialis anterior, rectus femoris, medial gastrocnemius, and the long head of the biceps femoris muscles of both the lower extremities were measured. [Results] The foot pressure of the dominant leg significantly decreased in the kinematic taping group. The muscle tone and stiffness in the rectus femoris muscle of the dominant and non-dominant leg, tibialis anterior muscle of the dominant leg, medial gastrocnemius muscle of the non-dominant leg, and the stiffness in the dominant leg significantly decreased. The muscle tone and stiffness generally increased in the sham taping group. However, no significant difference was observed between the 2 groups. [Conclusion] This study demonstrated that kinematic taping on flexible flat feet had positive effects of immediately reducing the abnormally increased foot pressure and the tone and stiffness in the lower extremity muscles.

Key words: Flexible flat feet, Kinematic taping, Muscle tone
\end{abstract}

(This article was submitted Nov. 30, 2015, and was accepted Jan. 16, 2016)

\section{INTRODUCTION}

Flat foot is a condition in which the medial longitudinal arch of a foot is chronically and abnormally collapsed ${ }^{1)}$. Flat foot is caused by the pathologic malalignment of the lower extremity (LE) joints ${ }^{2}$, abnormal muscle activity ${ }^{3}$, cartilage damage, and/or knee pain ${ }^{4}$. It should be appropriately managed because it may lead to increase in the tone and stiffness in the LE muscles $^{5)}$ and fatigue and cramps in the LE muscles and feet due to overuse ${ }^{2)}$. For flexible flat foot, surgical treatments are hardly preferred ${ }^{1}$, and activity modifications, weight loss, orthotic management, immobilization, and footwear modifications are used as initial treatment options in adults ${ }^{2}$. Most treatments include supporting the overstretched plantar fascia and the weakened medial longitudinal arch.

Among physical therapy interventions for flat foot, taping is widely used as an effective method that reduces foot pronation and abnormally increased LE muscle activity ${ }^{3,6}$. Kinematic taping used in the present study is an intervention in which elastic tapes are stretched $33 \%$ before being attached ${ }^{7}$.

Most previous studies have described the effects of flat foot treatment, which include improved height of the medial longitudinal arch and activity of the LE muscles ${ }^{3,8)}$. However, no study has examined the effects of taping on tone and

*Corresponding author. Gi-Mai Um (E-mail: ukm2000@hanmail.net)

(C)2016 The Society of Physical Therapy Science. Published by IPEC Inc.

This is an open-access article distributed under the terms of the Creative Commons Attribution Non-Commercial No Derivatives (by-nc-nd) License $<$ http://creativecommons.org/licenses/by-nc-nd/4.0/>. 
stiffness in the LE muscles.

Therefore, this study aimed to analyze the immediate effects of kinematic taping on the tone and stiffness in the muscles of both LEs in subjects with flat feet, and to provide basic clinical data and taping methods that can be effectively used in clinical practice.

\section{SUBJECTS AND METHODS}

In the present study, each subject was instructed to stand upright on a global posture system ([GPS] 400, Redbalance, Italy) while looking straight ahead, and the subject's soles were filmed using the camera of the GPS. Among these subjects, 30 were selected for the study. The selected subjects were confirmed to have flat feet either because the ratio of the shortest length of the concave region of their foot to the widest length of the ball of their foot, measured using the GPS program, exceeded 0.37 (pixel/pixel) ${ }^{9}$ ) and/or because their Marie's line ${ }^{10,11)}$ fell medial to the line of the medial sole. Marie's line refers to the line that connects the center of the third metatarsal bone to the center of the rear foot. Study subjects were selected only from among those who understood the context of the present study and agreed to participate. The present study was approved by the research ethics committee of Namseoul University of Cheonan.

The study subjects were randomly assigned either to the kinematic taping group (KT group), which included 15 subjects, 4 men and 11 women, or to the sham taping group (ST group), which included 15 subjects, 10 men and 5 women. The differences in the tone and stiffness in both LE muscles between the 2 groups were examined. The KT group's mean age was $20.2 \pm 0.7$ years, mean weight was $61.4 \pm 10.7 \mathrm{~kg}$, and mean height was $166.2 \pm 8.0 \mathrm{~cm}$; right leg of all the group members was dominant. The ST group's mean age was $20.5 \pm 1.1$ years, mean weight was $64.4 \pm 14.8 \mathrm{~kg}$, and mean height was 164.3 $\pm 8.1 \mathrm{~cm}$; right leg of all the group members was dominant. To test the homogeneity of the study groups, independent t-tests were conducted to analyze the general characteristics, foot pressure, muscle tone, and stiffness in the study subjects; the results showed no significant differences between the 2 groups.

For the measurement of muscle tone and stiffness, the highly reliable Myoton ${ }^{\circledR}$ PRO device (Myoton AS, Estonia) was used $^{12)}$. The muscles that were measured included the rectus femoris (RF), tibialis anterior (TA), medial gastrocnemius (MG), and the long head of the biceps femoris (BF) of both sides ${ }^{5)}$. Before measurement, skin markers were symmetrically placed on the highest points of the muscle bellies of individual muscles. The RF and TA muscles were measured in the supine position, and the MG and BF muscles were measured in the prone position. For the measurement, the tip of the measurement device was vertically placed on the skin marker with the muscle in relaxed condition, and the values were measured through 5 resonances ${ }^{5}$. All the measurements were conducted in independent quiet spaces with an average indoor temperature of $23{ }^{\circ} \mathrm{C}$.

In the taping intervention method, 5-cm wide elastic tapes (Benefact, Nippon Sigmax Co. Ltd., Japan) were applied to the posterior shin muscle and the transverse arch of both legs. Although the study subjects were educated to remove the tapes if they had severe itching or skin pulling, none of them experienced such symptoms. To attach the tapes in the KT group, the required tape length for each subject was measured and cut to three-quarters of that length. The tape for the posterior shin muscle was stretched and attached beginning from the transverse arch, passing the inside ankle, going diagonally cross the tibia bone, and reaching the lateral epicondyle of the femur. The tape for the transverse arch was attached when the study subject had made a transverse $\operatorname{arch}^{7}$. On taping, no stretch tension was applied to the origin and insertion of each tape. In the ST group, a tape was attached from the medial malleolus of the tibia to the lateral epicondyle of the femur without any ankle joint movement or tape stretch. All taping interventions were applied once for 24 hours.

All data were encoded and analyzed using the Statistical Packages for the Social Sciences WIN program (ver. 21). After interventions, independent t-tests were used to examine the differences in the tone and stiffness in the LE muscles in each study group, and paired t-tests were used to compare the effects of intervention between the 2 groups. The statistically significance level for all groups was set at $\mathrm{p}<0.05$.

\section{RESULTS}

After taping, the foot pressure in the dominant leg of the KT group significantly decreased $(\mathrm{p}<0.05)$. Except for the muscle tone and stiffness in the non-dominant leg, the tone and stiffness in all the measured muscles decreased. In particular, the tone and stiffness in the RF muscle of the dominant leg and non-dominant leg, TA muscle of the dominant leg, MG muscle of the non-dominant leg, and the stiffness in the dominant leg significantly decreased $(\mathrm{p}<0.05)$. In the ST group, muscle tone and stiffness generally increased. In particular, the tone of the BF and MG muscles of the non-dominant leg and the stiffness in the BF muscle of the non-dominant leg significantly increased $(\mathrm{p}<0.05)$. There were significant differences in the foot pressure of the dominant leg between the 2 groups $(\mathrm{p}<0.05)$ (Table 1$)$. 
Table 1. Changes in foot pressure and muscle tone and stiffness in each taping group

\begin{tabular}{lllcccc}
\hline \multirow{2}{*}{ Variable } & & \multirow{2}{*}{ Side } & \multicolumn{2}{c}{ Kinematic taping group } & \multicolumn{2}{c}{ Sham taping group } \\
\cline { 3 - 6 } Foot pressure & & & \multicolumn{1}{c}{ Before } & After & Before & After \\
(pixel/pixel) & & DL & $0.4 \pm 0.0$ & $0.4 \pm 0.0^{*}$ & $0.4 \pm 0.0$ & $0.4 \pm 0.0^{*}$ \\
& Muscle & NDL & $0.4 \pm 0.0$ & $0.4 \pm 0.0$ & $0.4 \pm 0.0$ & $0.4 \pm 0.0$ \\
Rectus & tone (Hz) & NDL & $13.9 \pm 0.3$ & $12.8 \pm 0.2^{*}$ & $13.6 \pm 0.2$ & $13.9 \pm 0.3$ \\
femoris & Stiffness & DL & $237.0 \pm 10.3$ & $183.4 \pm 11.1^{*}$ & $222.2 \pm 9.4$ & $237.9 \pm 10.5$ \\
& (N/m) & NDL & $233.4 \pm 10.0$ & $189.2 \pm 14.8^{*}$ & $219.6 \pm 9.1$ & $232.7 \pm 9.7$ \\
& Muscle & DL & $18.4 \pm 0.6$ & $16.5 \pm 0.6^{*}$ & $18.2 \pm 0.5$ & $18.7 \pm 0.5$ \\
Tibialis & tone (Hz) & NDL & $18.1 \pm 0.6$ & $17.4 \pm 0.5$ & $17.9 \pm 0.5$ & $18.1 \pm 0.6$ \\
anterior & Stiffness & DL & $386.6 \pm 21.0$ & $323.4 \pm 21.6^{*}$ & $369.6 \pm 16.3$ & $370.8 \pm 12.8$ \\
& (N/m) & NDL & $374.6 \pm 21.8$ & $354.3 \pm 17.1$ & $363.8 \pm 13.7$ & $366.8 \pm 15.8$ \\
Medial & Muscle & DL & $15.7 \pm 0.4$ & $15.0 \pm 0.4$ & $15.3 \pm 0.3$ & $16.1 \pm 0.3^{*}$ \\
gastrocne- & tone (Hz) & NDL & $15.7 \pm 0.4$ & $14.6 \pm 0.4^{*}$ & $15.2 \pm 0.4$ & $15.1 \pm 0.3$ \\
mius & Stiffness & DL & $268.0 \pm 10.9$ & $249.8 \pm 8.2^{*}$ & $258.8 \pm 6.9$ & $265.2 \pm 7.4$ \\
& (N/m) & NDL & $269.7 \pm 10.0$ & $242.7 \pm 9.2^{*}$ & $248.8 \pm 8.9$ & $262.1 \pm 8.3$ \\
Biceps & Muscle & DL & $15.0 \pm 0.4$ & $14.7 \pm 0.6$ & $15.0 \pm 0.2$ & $15.6 \pm 0.4$ \\
femoris & tone (Hz) & NDL & $14.7 \pm 0.4$ & $15.0 \pm 0.3$ & $14.6 \pm 0.3$ & $15.4 \pm 0.4^{*}$ \\
(long head) & Stiffness & DL & $254.8 \pm 10.7$ & $251.2 \pm 18.8$ & $250.8 \pm 8.6$ & $264.2 \pm 11.9$ \\
& (N/m) & NDL & $239.3 \pm 12.5$ & $261.8 \pm 11.9^{*}$ & $243.4 \pm 9.0$ & $259.7 \pm 11.9^{*}$ \\
\hline
\end{tabular}

Values are means \pm standard error, DL: dominant leg, NDL: non-dominant leg

*Significant difference between before and after the taping intervention in each group $(\mathrm{p}<0.05)$

*Significant difference between the kinematic taping group and sham taping group $(\mathrm{p}<0.05)$

\section{DISCUSSION}

The present study was conducted to identify the effects of KT on the tone and stiffness in the LE muscles of subjects with flexible flat feet.

Flat foot is associated with knee pain and medial tibiofemoral cartilage damage, and the associated foot pronation and lower leg internal rotation in standing positions could be due to increased postural stress in the tibiofemoral and patellofemoral tissues ${ }^{4}$. Um et al. ${ }^{5}$ suggested that the tone and stiffness in the TA and MG muscles of the dominant leg in subjects with flexible flat feet were significantly higher than those in subjects with normal feet.

In this study, after applying KT, the foot pressure in the dominant leg significantly decreased, as did the muscle tone and stiffness in most muscles except BF. Therefore, KT could be an effective method to improve abnormally increased muscle tone and stiffness and the foot pronation described in previous studies on flat foot ${ }^{4,5)}$. In addition, after applying ST, the tone and stiffness in the LE muscles increased, indicating that incorrect taping methods could adversely affect muscle tone and stiffness.

Franettovich et al. ${ }^{3)}$ suggested that when nonelastic taping was applied to flat foot, the muscle activity of the tibialis posterior, peroneus longus, and TA during walking decreased by $33 \%, 30 \%$, and $13 \%$, respectively, which was similar to the results of the present study. The results of the present study indicated that KT could affect the tone and stiffness not only of the muscles around the ankle but also of the thigh muscles. However, the stiffness in the muscles of the non-dominant leg of the KT group significantly increased. Because the non-dominant leg is used to support body weight in the standing position, the muscle tone and stiffness in the BF could have temporarily increased after KT to compensate for the decreased tone and stiffness in other LE muscles.

The method of taping used in the present study, in which each tape for the posterior shin muscle was stretched and attached beginning from the transverse arch, passing the inside ankle, going diagonally across the tibia bone, and reaching the lateral epicondyle of the femur, unlike in previous studies in which taping was applied to the muscles around the ankle ${ }^{3,8)}$, is thought to have reduced the tone and stiffness in the muscles around the ankle. Therefore, the taping method used in the present study could be effectively used to manage flat foot or knee pain and flat foot. Additionally, KT can be recommended for self-taping during flat foot treatment at clinics because it can be easily applied and taught through patient education. However, such cases would require attention because wrong application of the tape may increase the abnormally increased tone and stiffness in the LE muscles of patients with flexible flat foot.

Through the present study, it was observed that application of KT to flexible flat feet was an effective intervention method for immediately reducing abnormally increased foot pressure and tone and stiffness in the LE muscles. 


\section{REFERENCES}

1) Neumann DA: Kinesiology of the musculoskeletal system (Foundations for rehabilitation). St Louis: Mosby, 2010.

2) Lee MS, Vanore JV, Thomas JL, et al. Clinical Practice Guideline Adult Flatfoot Panel: Diagnosis and treatment of adult flatfoot. J Foot Ankle Surg, 2005, 44: 78-113. [Medline] [CrossRef]

3) Franettovich MM, Murley GS, David BS, et al.: A comparison of augmented low-dye taping and ankle bracing on lower limb muscle activity during walking in adults with flat-arched foot posture. J Sci Med Sport, 2012, 15: 8-13. [Medline] [CrossRef]

4) Gross KD, Felson DT, Niu J, et al.: Association of flat feet with knee pain and cartilage damage in older adults. Arthritis Care Res (Hoboken), 2011, 63: 937-944. [Medline] [CrossRef]

5) Um GM, Wang JS, Park SE: An analysis on muscle tone of lower limb muscles on flexible flat foot. J Phys Ther Sci, 2015, 27: 3089-3092. [Medline] [CrossRef]

6) Lange B, Chipchase L, Evans A: The effect of low-Dye taping on plantar pressures, during gait, in subjects with navicular drop exceeding $10 \mathrm{~mm}$. J Orthop Sports Phys Ther, 2004, 34: 201-209. [Medline] [CrossRef]

7) Langendoen J, Sertel K: Kinesiology Taping. Robert Rose Inc., 2014.

8) Franettovich M, Chapman A, Vicenzino B: Tape that increases medial longitudinal arch height also reduces leg muscle activity: a preliminary study. Med Sci Sports Exerc, 2008, 40: 593-600. [Medline] [CrossRef]

9) Global posture system 400 manual.

10) Clarke HH: Application of measurement to Health and Physical Education, 5th ed., 1976.

11) Kim MK, Lee YS: Kinematic analysis of the lower extremities of subjects with flat feet at different gait speeds. J Phys Ther Sci, 2013, 25: 531-533. [Medline] [CrossRef]

12) Aird L, Samuel D, Stokes M: Quadriceps muscle tone, elasticity and stiffness in older males: reliability and symmetry using the MyotonPRO. Arch Gerontol Geriatr, 2012, 55: e31-e39. [Medline] [CrossRef] 